\title{
The Subcellular Organization of Cortactin in Hippocampus
}

\author{
Bence Racz ${ }^{1}$ and Richard J. Weinberg ${ }^{1,2}$ \\ ${ }^{1}$ Department of Cell and Developmental Biology and ${ }^{2}$ Neuroscience Center, University of North Carolina, Chapel Hill, North Carolina 27599
}

\begin{abstract}
Spines may undergo rapid, activity-dependent changes in shape and size, reflecting reorganization of the actin cytoskeleton. This remodeling is implicated in development and also in the late phase of long-term potentiation. However, the cellular mechanisms that convert activity into morphological change remain poorly understood, and little is known about the anatomical distribution of the actin-regulating proteins that mediate this remodeling. Using immunocytochemistry, we demonstrate here that cortactin (a protein implicated in actin filament nucleation, branching, and stabilization) is concentrated in hippocampal spines, where it colocalizes with F-actin. Cortactin has a Shank-binding domain; recent studies report that synaptic activity may trigger actin remodeling via this interaction with Shank. However, our immunogold electron microscopic data show that cortactin concentrates within the spine core, $100-150$ $\mathrm{nm}$ away from the postsynaptic density (PSD); only a small fraction of the cortactin in spines lies adjacent to the PSD. These data suggest that the adult dendritic spine contains two functional pools of cortactin: a large pool in the spine core that may help to mediates changes in spine shape and a small synaptic pool that may modify the PSD in response to synaptic activity.
\end{abstract}

Key words: actin; dendritic spine; cytoskeleton; immunogold; electron microscopy; $\alpha$-actinin

\section{Introduction}

The dendrites of excitatory neurons in the mammalian forebrain are covered with spines, the principal loci of synaptic processing (Ramón y Cajal, 1909). Typically, a single glutamatergic bouton makes synaptic contact onto the spine head (Harris, 1999). Immediately beneath this synaptic apposition lies the postsynaptic density (PSD), an electron-dense matrix that contains glutamate receptors, signal transduction molecules, and adaptor proteins (Kennedy, 2000; Sheng, 2001). Accumulating evidence shows that dendritic spines may change their shape in response to glutamate, especially via activation of NMDA receptors (Halpain, 2000; Segal and Andersen, 2000). Besides its role in development (Maletic-Savatic et al., 1999), spine remodeling has been associated with synaptic plasticity in the mature brain (Yuste and Bonhoeffer, 2001; Trachtenberg et al., 2002). Although cytoskeletal reorganization is coupled to glutamatergic synaptic activity, the mechanisms underlying this coupling remain unclear.

Electron micrographs reveal an intimate linkage between the PSD and a network of actin-based filaments that may span the length of the spine (Landis and Reese, 1983; Fifkova and Morales, 1992; Matus, 2000). Such images suggest a direct connection between synaptic activity and changes in spine geometry. The biochemical machinery underlying this connection is not yet understood, although $\alpha$-actinin, a protein concentrated at the PSD that can bind to both actin and the NMDA receptor, has been impli-

Received May 28, 2004; revised Aug. 19, 2004; accepted 0ct. 5, 2004.

This work was supported by the National Institutes of Health-National Institute of Neurological Disorders and Stroke. B.R. had the idea and performed the experiments. R.J.W. provided support and guided experiments and analysis. We thank Alain Burette, Michael Ehlers, Marcello Melone, Carol Otey, and Juli Valtschan off for comments on this manuscript and Susan Grand and Kris Phend for technical assistance.

Correspondence should be addressed to Dr. Richard J. Weinberg, Department of Cell and Developmental Biology, CB \#7090, University of North Carolina, Chapel Hill, NC 27599. E-mail rjw@med.unc.edu.

DOI:10.1523/JNEUROSCI.2080-04.2004

Copyright $\odot 2004$ Society for Neuroscience $\quad 0270-6474 / 04 / 2410310-08 \$ 15.00 / 0$ cated (Wyszynski et al., 1998). Spine remodeling can also be triggered by activation of metabotropic glutamate receptors (Vanderklish and Edelman, 2002). This effect may be mediated via Shank, a PSD protein that can link to both the NMDA receptor and the metabotropic receptor (Naisbitt et al., 1999; Tu et al., 1999; Sala et al., 2001).

For a PSD scaffolding protein to modify spine shape, it must affect the cytoskeleton. It is therefore intriguing that Shank interacts with the actin-binding protein cortactin (Naisbitt et al., 1999). Cortactin, an $\sim 80 \mathrm{kDa}$ protein found throughout the animal kingdom, contains an Arp 2/3-binding site near its $\mathrm{N}$ terminal, five to six tandem repeats of an actin-binding domain, and a proline-rich region, followed by an Src homology 3 domain at its C terminal (Kanner et al., 1990; Wu and Parsons, 1993). Thus, cortactin can bind to actin while simultaneously activating the actin-nucleating Arp2/3 complex (May, 2001; Weaver et al., 2001; Daly, 2004). Cortactin localizes to lamellopodia, filopodia, and membrane ruffles at the leading edge of migrating fibroblasts, where it colocalizes with F-actin and the Arp2/3 complex; this association is thought to mediate actin-based motility (Weed et al., 2000; Weed and Parsons, 2001; Krueger et al., 2003). Recent studies in cultured neurons suggest that cortactin may play an analogous role in brain (Hering and Sheng, 2003; Martinez et al., 2003). Despite this evidence implicating cortactin in the dynamic organization of dendritic spines, very little is known about its distribution in the intact brain.

We performed immunocytochemical experiments to find out whether cortactin is present in spiny neurons of the rat brain, and, if so, whether it concentrates in a location suited to transduce interactions between the synapse and the actin cytoskeleton.

\section{Materials and Methods}

Preparation of tissue. Experiments were performed on adult (3-5 months old) male Sprague Dawley rats from Charles River (Raleigh, NC). To 


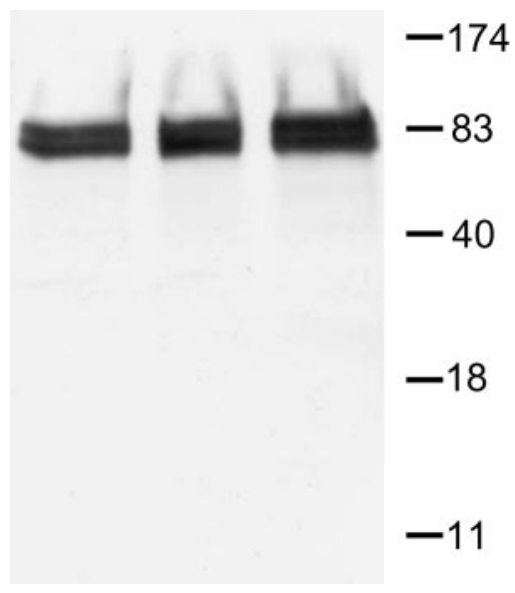

Figure 1. Western blot. A polyclonal antiserum generated against a recombinant protein corresponding to amino acids 309 - 499 of human cortactin detected two major protein bands, migrating at $\sim 80$ and $\sim 85 \mathrm{kDa}$ (left lane, cortex; middle lane, hippocampus; right lane, cerebellum). The molecular weights are in kilodaltons.

explore development, we also used material from postnatal day 14 (P14) and P21 rats. Animal housing and all experimental procedures were strictly in compliance with Institutional Animal Care and Use Committee guidelines. The animals were deeply anesthetized with pentobarbital $(60 \mathrm{mg} / \mathrm{kg}$, i.p.), then perfused intracardially through the left ventricle with $0.9 \% \mathrm{NaCl}$, followed by a mixture of depolymerized paraformaldehyde (PFA; $4 \%$ ) and glutaraldehyde $(0.5 \%)$ in $0.1 \mathrm{M}$ phosphate buffer (PB), pH 7.4 (for electron microscopy), or 1\% PFA (for light microscopy). Brains were removed and immersed in the same fixative for $2 \mathrm{hr} ; 50$ $\mu \mathrm{m}$ coronal sections were cut with a vibratome between -3.0 and $-6.0 \mathrm{~mm}$ caudal from bregma and processed for immunohistochemistry.

Antibodies. The primary antibodies used included polyclonal rabbit anti-cortactin $(0.4 \mu \mathrm{g} / \mathrm{ml}$; Santa Cruz Biotechnology, Santa Cruz, CA), monoclonal mouse anti-synaptophysin (clone SVP38, ascites fluid, 1:1000; Sigma, St. Louis, MO), polyclonal guinea pig anti-vesicular glutamate transporter 1 (VGLUT1; 1:2000; Chemicon, Temecula, CA), and monoclonal mouse anti- $\alpha$-actinin (clone EA-53, ascites fluid, 1:500; Sigma). Most of these antibodies are widely used and have been characterized in previous studies: for synaptophisin, see Jahn et al. (1985) and Wiedenmann and Franke (1985); for VGLUT1, see Fujiyama et al. (2001) and Todd et al. (2003); for $\alpha$-actinin, see Wyszynski et al. (1998). The cortactin antibody was the same used by Hering and Sheng (2003); to verify its specificity, we performed Western blot analysis. Briefly, proteins were separated by SDS-PAGE (10\%), transferred to Immobilon-P polyvinylidene difluoride membranes, and blocked with $5 \%$ fat-free dry milk (Carnation) in sample buffer (20 mM Tris, $137 \mathrm{~mm} \mathrm{NaCl}$, pH 7.6, and $0.1 \%$ Tween 20). Primary antibody was diluted 1:200 in blocking solution. Immune complexes were visualized with an ECL immunodetection kit (Amersham Biosciences, Piscataway, NJ); bands were recorded using an imaging station from Eastman Kodak (Rochester, NY), using ProSieve broad-range markers to determine molecular weights. As shown in Figure 1, the antibody recognized two bands in the $\sim 80-85$ $\mathrm{kDa}$ range in the cortex, hippocampus, and cerebellum.

Immunocytochemistry for confocal microscopy. Sections were blocked in $20 \%$ normal donkey serum (NDS; Jackson ImmunoResearch, West Grove, PA) in 0.05 m PBS, pH 7.4, then incubated in various combinations of primary antibodies for cortactin, $\alpha$-actinin, synaptophysin, and VGLUT1 in PBS containing 2\% NDS overnight at room temperature. After several washes, sections were incubated in secondary antibodies (anti-rabbit Cy3 for cortactin, anti-mouse FITC for synaptophysin and VGLUT1). Alexa Fluor-488 conjugated to phalloidin (Molecular Probes, Eugene, OR) was used for visualization of F-actin (Allison et al., 1998). For visualization of cell processes, we used the lipophilic dye 3,3'dioctadecyloxacarbocyanidine perchlorate (DiO; Molecular Probes), which infiltrates the plasma membrane, labeling even the finest neuronal processes (for details, see Burette et al., 2002). After several washes, sec- tions were mounted on glass slides, coverslipped in Vectashield (Vector Laboratories, Burlingame, CA) and examined with an SP2 laser scanning confocal microscope (Leica, Nussloch, Germany). Optical sections acquired with a Plan Apo $63 \times$ oil objective (numerical aperture, 1.4) were scanned in a $1024 \times 1024$ pixel format. Images stored as RGB TIFF images were digitally processed with Adobe Photoshop CS (version 8.0; Adobe Systems, Mountain View, CA). Each image was cropped and sharpened with unsharp masking; contrast, brightness, tonal range, and color balance were edited. Each processing step was applied uniformly to the entire image.

Immunocytochemistry for electron microscopy. For immunoperoxidase staining, floating sections were treated for $30 \mathrm{~min}$ in $1 \%$ sodium borohydride in PB to quench free aldehyde groups. The sections were incubated in 20\% NDS for 30 min to suppress nonspecific binding and then for $12 \mathrm{hr}$ in anti-cortactin antibody, along with $2 \%$ NDS. Sections were incubated in biotinylated anti-rabbit IgG (Jackson ImmunoResearch) for $2 \mathrm{hr}$. After several washes in PBS, sections were then incubated in Extravidin peroxidase (1:5000; Sigma) for $1 \mathrm{hr}$. The immunopositive structures were visualized with $3,3^{\prime}$-diaminobenzidine tetrahydrochloride (DAB). Sections were processed as described above in control experiments, omitting primary antibody from the incubation solution. In such sections, no immunostaining was observed. Some immunoperoxidasestained sections were prepared for light microscopy; these were mounted on gelatin-coated glass slides, dehydrated in ascending ethanol series, cleared with xylene, and coverslipped with DPX (Sigma) mountant.

For preembedding gold, sections were incubated together with those for immunoperoxidase staining up to the secondary antibody stage. After rinses in PBS, sections were incubated in goat-anti rabbit IgG coupled to $1.4 \mathrm{~nm}$ gold particles (1:30; Nanoprobes, Yaphank, NY) for $3 \mathrm{hr}$ at room temperature and rinsed in PBS. Sections were washed in $0.1 \mathrm{M} \mathrm{Na}$ acetate (to remove phosphate and chloride ions), followed by silver enhancement with Intense S-EM (Amersham Biosciences).

Sections for electron microscopy were postfixed in $0.5-1 \%$ osmium tetroxide in $0.1 \mathrm{M} \mathrm{PB}$ for 35-45 min and contrasted with $1 \%$ uranyl acetate for $1 \mathrm{hr}$. After dehydration in ascending ethanol series and propylene oxide, sections were infiltrated with Epon/Spurr resin (Electron Microscopy Sciences, Hatfield, PA) and flat-mounted between sheets of Aclar within glass slides. Seventy-nanometer sections were cut, mounted on 200 mesh copper grids, contrasted with uranyl acetate and Sato's lead, and examined in a Philips (Hillsboro, OR) Tecnai electron microscope at $80 \mathrm{kV}$; images were collected with a Gatan (Pleasanton, CA) 12-bit $1024 \times 1024$ CCD camera.

Quantitative analysis of the immunogold reaction. Electron micrographs were taken from randomly selected fields, focusing on the proximal and middle regions of CA 1 stratum radiatum, extending out to $\sim 250$ $\mu \mathrm{m}$ from the cell layer. To determine the relative density of cortactin in distinct subcellular compartments, axon terminals, dendritic shafts, spines, and pyramidal cell nuclei were identified; gold particles within their cytoplasm were counted, and the areas were measured. Both labeled and unlabeled profiles were included in the analysis. To determine background, we calculated labeling over pyramidal cell nuclei (because nuclei did not stain for cortactin in LM material). For details on measuring positions of gold particles, see Valtschanoff and Weinberg (2001). Membrane perimeters and profile areas were measured using the area calculator plug-in of ImageJ version 1.29 software (National Institutes of Health, Bethesda, MD). This plug-in measures area, total area, and average area (for additional information, see http://rsb.info.nih.gov/ij/ plugins/area.html). Particle densities for somatic, dendritic, and spine cytoplasm were computed and compared with nonspecific labeling, using a two-sided $t$ test. Microsoft Excel, Kaleidagraph (Synergy Software, Reading, PA), CricketGraph (Computer Associates, Islandia, NY), and Data Desk (Data Descriptions, Ithaca, NY) were used to generate graphs and to compute statistics.

\section{Results \\ Light microscopy}

Cortactin immunostaining was seen in gray matter throughout the brain, sparing white matter. Staining was prominent in several areas rich in dendritic spines, including the cerebellar cortex, 

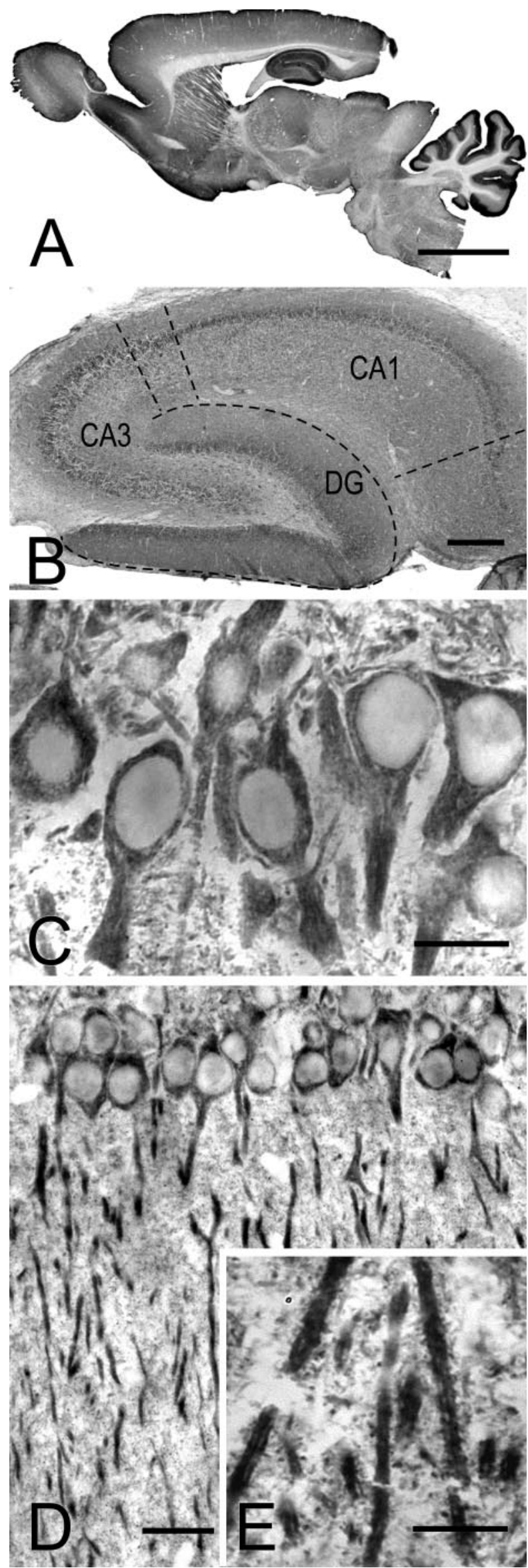

olfactory bulb, striatum, cerebral neocortex, and hippocampal formation (Fig. 2A). Within the hippocampal formation, staining was dense in the neuropil of Ammon's horn and the molecular layer of the dentate gyrus (Fig. $2 B$ ). For this study, we focused our attention on CA1, the spiny pyramidal neurons of which have been especially well studied. Immunoreaction was present in CA1 pyramidal neurons, labeling their cell bodies and apical dendrites but sparing the nucleus; somatic staining was organized into small patches (Fig. 2C). No obvious glial staining was observed. Proximal dendrites contained abundant immunoperoxidase reaction product, often associated with filamentous structures (Fig. 2C,D). High-magnification views revealed punctate staining in the stratum radiatum (Fig. $2 E$ ), suggesting that cortactin may be concentrated in dendritic spines and/or terminals.

To elucidate the organization of cortactin in the neuropil, we performed high-resolution confocal microscopy on material double labeled for cortactin and presynaptic markers. Cortactin in the stratum radiatum was punctate; these puncta were frequently adjacent to synaptophysin-immunopositive puncta. Cortactin puncta often showed small regions of overlap with synaptophysin puncta (Fig. 3A, arrows), but seldom was extensive overlap detected, suggesting that cortactin concentrates in postsynaptic structures. The same pattern was also seen after double labeling with cortactin and VGLUT1, a marker for glutamatergic terminals (Fig. 3B) (Fremeau et al., 2001; Kaneko and Fujiyama, 2002).

These results, along with previous data from cultured neurons, suggested that cortactin in the hippocampus might concentrate in dendritic spines. To clarify the issue, we used the lipophilic dye $\mathrm{DiO}$ to label the dendritic plasma membrane. This experiment confirmed that cortactin concentrates in dendritic spines in the adult brain (Fig. 3C, arrowheads). Because cortactin has an F-actin-binding domain, we wondered whether it colocalized with actin in specific subcellular compartments. We therefore performed double labeling with phalloidin and found massive dendritic colocalization, especially in spines (Fig. 3D) (cf. Capani et al., 2001a,b).

These LM data show that cortactin is expressed at high levels in spiny neurons and suggest that it concentrates along with actin in dendritic spines.

\section{Electron microscopy}

To gain a better understanding of its subcellular organization, we performed immmunoelectron microscopy for cortactin. In cell bodies of CA1 pyramidal neurons, the electron-dense immunoperoxidase reaction product was mostly associated with rough endoplasmatic reticulum (Fig. 4A). Most dendritic shafts contained patches of reaction product, but staining was markedly denser in spines (Fig. $4 B, C$, asterisks). Reaction product often filled spines, although in some cases it was attenuated or absent

\section{$\leftarrow$}

Figure 2. Immunoperoxidase staining for cortactin. A, Low-magnification view of a parasagittal section of adult rat brain. Staining is especially prominent in the cerebellar cortex and hippocampus and is also strong in other brain regions rich in dendritic spines. B, Micrograph showing the hippocampal formation. Staining is conspicuous in the molecular layer of the dentate gyrus (DG) and in the neuropil of CA1 (dashed lines mark borders of the main regions within the hippocampal formation). C, D, CA1 of hippocampus, showing stained somata and apical dendrites of pyramidal neurons. The detail in C reveals patchy staining in the somatodendritic compartment, surrounding immunonegative nuclei. E, High-magnification view of the stratum radiatum reveals intensely labeled dendrites; puncta in the neuropil may correspond to dendritic spines. Scale bars: $A, 5 \mathrm{~mm} ; B, 500 \mu \mathrm{m} ; C, E, 25 \mu \mathrm{m} ; D, 50 \mu \mathrm{m}$. 

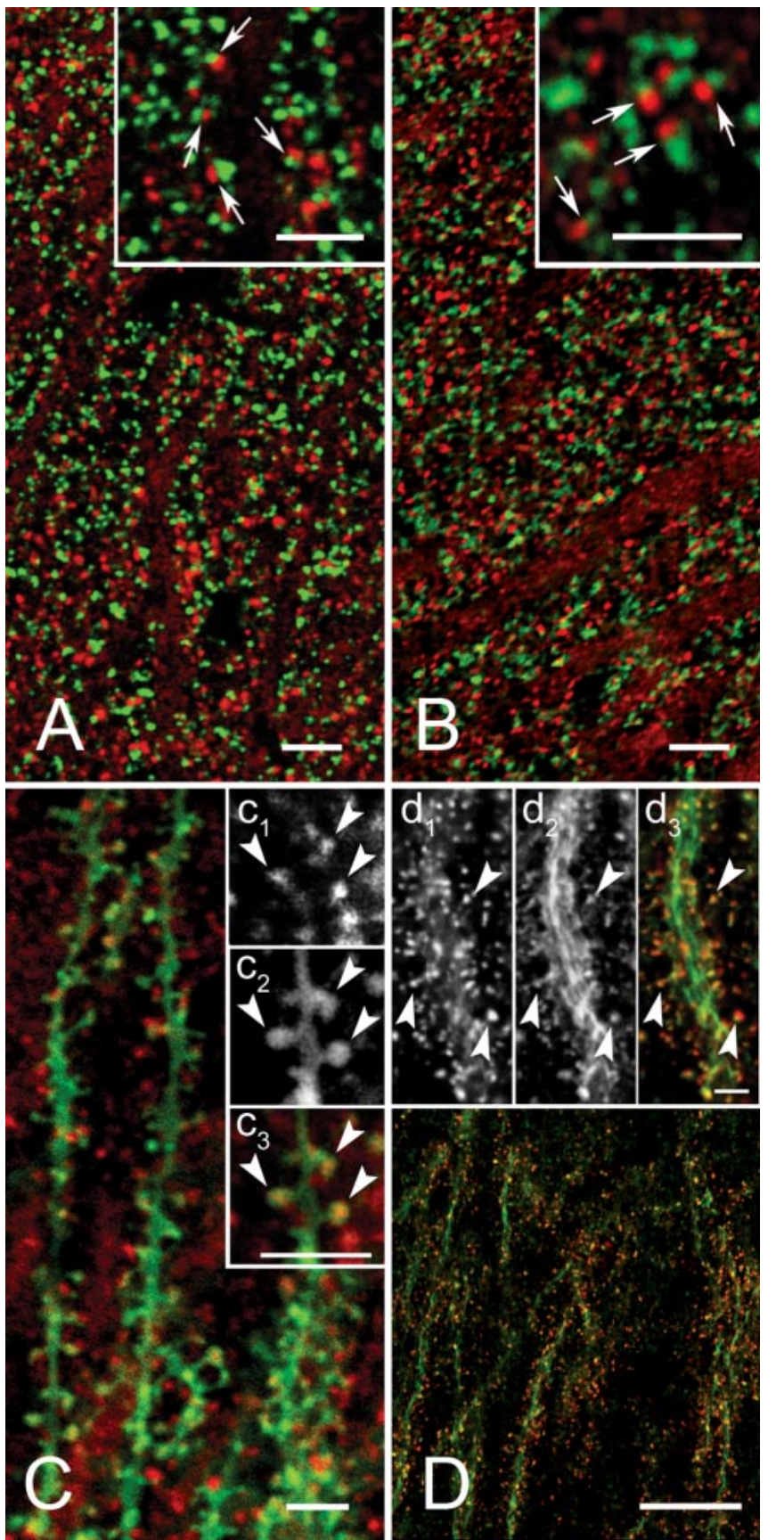

Figure 3. Subcellular distribution of cortactin in stratum radiatum of CA1. A, Confocal microscopy shows cortactin (red) and the presynaptic marker synaptophysin (green). The apposition of puncta immunopositive for the two markers (arrows in inset) suggests that cortactin may be postsynaptic. B, Double immunofluorescence with cortactin (red) and VGLUT1 (green). The pattern of punctate apposition suggests that cortactin is associated with glutamatergic synapses. C, Cortactin immunostaining was performed on material treated with the lipophilic dye Di0 (green), which stains the plasma membrane of scattered pyramidal cells. Inset, Cortactin puncta $\left(c_{1}\right)$ can be seen within Di0-stained $\left(C_{2}\right)$ spines (merge; $C_{3}$, arrowheads). D, Double fluorescence with cortactin (red) and phalloidin (green). Results suggest that cortactin localizes profiles also rich in actin. Inset, Arrowheads pointing to both cortactin-positive $\left(d_{1}\right)$ and phalloidin-positive $\left(\mathrm{d}_{2}\right)$ sspines. Scale bars: $A-C, 5 \mu \mathrm{m} ; D, 20 \mu \mathrm{m}$; insets, $5 \mu \mathrm{m}$.

from the region just beneath the PSD (Fig. $4 D, E$ ). Reaction product was not detected in axons and axon terminals. Thus, immunoperoxidase electron microscopy confirms our LM observation that cortactin concentrates in spines.

The above results suggest that cortactin in spines may lie away from the synaptic junction. However, these data do not allow critical examination of the question, because immunoperoxidase product may migrate away from the enzymatic site, often filling the profile and making it difficult to define the precise location of the antigen. To clarify this issue, we performed a set of experiments with immunogold labeling. We repeatedly tried postembedding techniques, but with little success; presumably the relevant epitope is destroyed or occluded by the preliminary histological processing. We therefore performed preembedding immunogold labeling, followed by silver intensification, to obtain more precise spatial localization of cortactin.

Numerous gold/silver particles coding for cortactin were seen in dendritic shafts of CA1 pyramidal neurons (Fig. 5A). Most of these particles were associated with microtubules, presumably representing a transport pool trafficking between spines and the cell body. Some dendritic labeling was not associated with microtubules; we speculate that this pool is coupled to actin filaments in the dendritic shaft, as hinted by phalloidin/cortactin double immunfluorescent labeling (Fig. 3D). In a number of cases, cortactin (presumably in association with actin filaments) concentrated in the general vicinity of asymmetric synaptic contacts onto dendritic shafts of putative interneurons (Fig. 5B).

Immunofluorescence and EM immunoperoxidase indicate that axons and axon terminals do not contain cortactin; however, after immunogold processing, occasional gold particles were seen in axon terminals (Fig. 5D, star). To assess whether these particles might represent unspecific labeling, we performed quantitative analysis of the density of immunogold labeling in different subcellular compartments. Nonspecific background labeling was calculated by measuring particle densities over the nuclei of pyramidal cells, because these were consistently immunonegative with immunofluorescence (see Materials and Methods). Labeling over axon terminals was not significantly above this background $(p>0.3)$ (Table 1). In contrast, dendritic shafts (8 times above background) and spines (15 times above background) contained significantly higher concentrations of gold particles than did nuclei $(p<0.001)$.

Labeling was especially prominent in the spines of pyramidal neurons. Gold particles in spines usually spared the immediate region of the PSD (Fig. 5C, arrows). Within spines, gold particles were often associated with electron-dense filamentous material (Fig. 5D,E), suggesting an interaction between cortactin and the cytoskeleton. In a number of cases, gold particles were seen in association with coated pits and vesicles in spines and dendritic shafts, although these profiles were uncommon.

Because cortactin has been found to increase spine size in cultured neurons, we wondered whether larger spines in the intact brain contain higher concentrations of cortactin. However, examining 236 randomly selected spine profiles, we found a weak negative correlation between labeling density and profile area $(r=-0.11)$. Random stochastic noise inherent in sampling from small profiles is likely to lead to underestimates of correlation. To reduce this noise, we examined correlations after grouping the data into 5, 10, or 20 bins of profile area and found correlations ranging from -0.43 to -0.50 . Because work on cultured neurons suggests that cortactin may be important for spine development, we speculated that cortactin density might be positively correlated with spine size during postnatal development. However, a similar weakly negative relationship was also seen in immature animals: in a sample of 102 profiles from $\mathrm{P} 21$ hippocampus, $r=$ -0.04 , and in a sample of 106 profiles from P14, $r=-0.18$; again, the magnitude of the correlation, but not its sign, increased with binning. 


\section{Distribution of cortactin within spines}

To establish the organization of cortactin within spines, we measured the distances of gold particles from the outer leaflet of the postsynaptic membrane, as described previously (Valtschanoff and Weinberg, 2001). Consistent with hints from the DAB material (Fig. 4D,E), cortactin labeling concentrated away from the PSD, in the cytoplasmic core of the spine, with a peak labeling density $\sim 100-150 \mathrm{~nm}$ away from the synaptic membrane (Fig. $6 A, \bigcirc$ ). This result must be treated cautiously: preembedding techniques may fail to detect antigen within the dense protein matrix of the PSD (Lorincz et al., 2002). This possibility concerned us because some biochemical studies (Husi et al., 2000; Peng et al., 2004) have reported cortactin in the PSD, although others failed to detect cortactin (Walikonis et al., 2000; Yoshimura et al., 2004).

To control for the possibility that methodological limitations might have prevented detection of cortactin in the PSD, we used the same preembedding technique to detect $\alpha$-actinin, an actinbinding protein previously demonstrated with postembedding techniques to concentrate within the PSD (Wyszynski et al., 1998). We found that particles coding for $\alpha$-actinin concentrated $50-70 \mathrm{~nm}$ from the PSD, significantly closer than cortactin to the synaptic plasma membrane $(p<0.001)$ (Fig. $6 A, \mathbf{\Delta})$. Examining these data further, we found that $9 \%$ of the particles coding for $\alpha$-actinin (17 of 188) lay $\leq 30 \mathrm{~nm}$ from the postsynaptic membrane (a region clearly within the PSD), whereas none of the cortactin particles $(0$ of 122) lay in this region. It is thus unlikely that methodological limitations prevented detection of cortactin within the PSD. However, $\sim 10 \%$ of the gold particles were $<60 \mathrm{~nm}$ from the synaptic membrane, close enough to permit a direct interaction with binding partners within the PSD, especially proteins like Shank that lie in its inner layer (Valtschanoff and Weinberg, 2001). As an additional control, we examined the distribution of synaptophysin (Fig. 6C, $\triangle$ ), confirming that it was exclusively presynaptic, lying mainly $>100 \mathrm{~nm}$ from the synaptic membrane. Thus, our data indicate that the major pool of cortactin lies within the spine core and suggests that the modest fraction associated with the synapse lies at the cytoplasmic fringe of the PSD.

It still remains possible from the above data that cortactin accumulates close to the plasma membrane but away from the PSD. To test this possibility, we measured the distance of gold particles coding for cortactin to the spine plasma membrane (Fig. $6 B)$. The virtually complete absence of cortactin within $30 \mathrm{~nm}$ of the plasma membrane, in contrast to its accumulation $\sim 100-150$ $\mathrm{nm}$ away, confirmed that cortactin concentrates in the spine core.

\section{Discussion}

An accumulating body of evidence associates long-term synaptic plasticity in the hippocampus with changes in the morphology of dendritic spines (Segal and Andersen, 2000; Yuste and Bonhoeffer, 2001; Nikonenko et al., 2002; Harris et al., 2003), but the cellular mechanisms leading from activation of glutamate recep- tors to spine remodeling are not well understood. Spines are highly enriched in actin (Fifkova and Morales, 1992; Roelandse et al., 2003), and it has long been suspected that actin remodeling contributes to synaptic plasticity (Fifkova and Delay, 1982). Recent evidence demonstrates that interfering with actin polymerization can block long-term potentiation and thus confirms that changes in the actin cytoskeleton play an essential role in at least some forms of synaptic plasticity (Kim and Lisman, 1999; Halpain, 2000; Krucker et al., 2000; Fukazawa et al., 2003).

\section{Proteins that regulate actin remodeling}

The Arp2/3 complex, a key regulator of actin polymerization, is a likely component of spine remodeling (Machesky and Insall, 1998; Svitkina and Borisy, 1999; Meyer and Feldman, 2002). Arp2/3 functions efficiently only when activated; its best-known activator is the Wiskott-Aldrich syndrome protein (WASP) (Mullins, 2000), but cortactin can also activate Arp2/3-mediated actin assembly (Uruno et al., 2001; Weaver et al., 2001). However, cortactin has a special branch-stabilizing activity that WASP lacks (Uruno et al., 2003). Thus, cortactin is likely to have different cellular functions than the WASP protein family.

Complete stabilization of the actin network would prevent the spine from functioning as a plastic receptive unit (Kasai et al., 2003). Cortactin in model systems provides only partial stabilization, thus permitting continued flexibility or growth (Uruno et al., 2003); we suggest that cortactin plays an analogous role in dendritic spines. Furthermore, cortactin moves from the spine into the dendritic shaft after activation of NMDA receptors (Hering and Sheng, 2003), a stimulus previously found to stabilize spine morphology (Star et al., 2002). Intriguingly, profilin, an- 

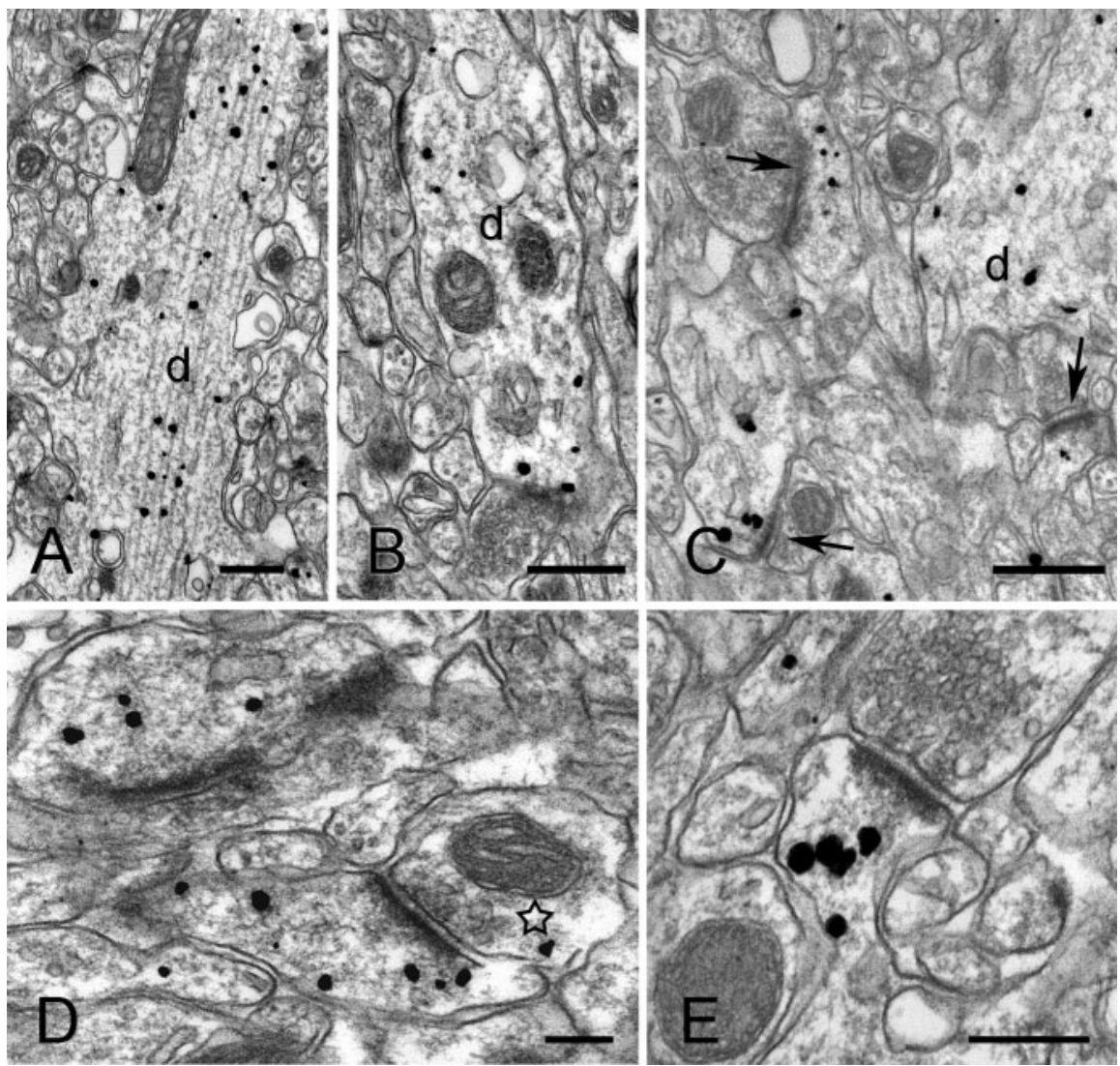

Figure 5. Preembedding immunogold labeling for cortactin in stratum radiatum of CA1. $A, B$, Electron micrographs show immunopositive dendrites (d). Most of the silver-enchanced gold particles visible in the apical dendrite in $A$ are associated with microtubules. Gold particles in the dendrite of a possible interneuron $(B)$ concentrate close to asymmetric synaptic appositions. $C$, Low-magnification view of synaptic neuropil. Arrows point to three immunolabeled spines; d marks an immunopositive dendritic shaft. $D, E$, High-magnification views showing immunopositive spines. Gold particles extend deep into the spine core (star in $D$ indicates axon terminal with a gold particle). Scale bars: $A-C, 0.5 \mu \mathrm{m} ; D, E, 0.2 \mu \mathrm{m}$.

Table 1. Densities of gold particles coding for cortactin in different subcellular compartments

\begin{tabular}{lc}
\hline & Density $\left(\right.$ particles $\left./ \mu \mathrm{m}^{2}\right)$ \\
\hline Nucleus & $0.66 \pm 0.05(n=11)$ \\
Axon terminal & $1.13 \pm 0.45(n=30)$ \\
Dendritic shaft & $5.25 \pm 0.50(n=14)^{*}$ \\
Spine & $9.92 \pm 1.25(n=44)^{*}$
\end{tabular}

Randomly selected profiles identifiable as axon terminals, dendritic shafts, or spines were included in the analysis, regardless of whether they were immunopositive. All data were collected from CA1 stratum radiatum, $4.0 \mathrm{~mm}$ caudal to bregma. To assess noise, nuclear profiles from the stratum pyramidale were also examined. Only dendritic shafts and spines showed significant differences from the nuclear background. ${ }^{*} p<0.001$; two-sided $t$ test (mean $\pm \mathrm{SE} ; n=$ number of profiles).

other regulator of actin polymerization (Finkel et al., 1994), moves into the spine head after NMDA receptor activation (Ackermann and Matus, 2003). Because profilin acts to stabilize spine morphology, we suggest that the NMDA receptor-mediated exchange of profilin for cortactin may represent an activitydependent switch regulating actin stability.

\section{Spine morphology and cortactin}

Recent experiments in cultured neurons (Hering and Sheng, 2003) suggest a role for cortactin in spine development. Cortactin can bind to Shank, a scaffold protein previously shown to increase spine size. However, this association is apparently not the primary mediator of the cortactin effect, because Shank overexpression increased spine head thickness, whereas cortactin over- expression increased spine length; furthermore, cortactin mutants unable to bind to Shank also increase spine length (Hering and Sheng, 2003). Our findings extend this work by showing that cortactin concentrates in spines in the mature brain. Unexpectedly, our data suggest that cortactin is more concentrated in small spines, at variance with results from cultured neurons (Hering and Sheng, 2003). Cortactin might play functionally different roles in developing and mature neurons, but we also saw a negative correlation in spines from the immature hippocampus. Overexpression experiments may not reflect the physiological function of cortactin in vivo; alternatively, one could speculate that small spines containing high concentrations of cortactin have been "marked" for subsequent enlargement. Although purely observational, our results are consistent with Kasai's model (Kasai et al., 2003; Matsuzaki et al., 2004) in that small spines, presumably available for plasticity-dependent remodeling, contain more cortactin than large, presumably stable spines.

\section{Distribution of cortactin within spines}

Our data show that cortactin concentrates at a considerable distance $(\sim 100-150$ $\mathrm{nm})$ from the synapse, suggesting that it interacts with actin in the spine core, away from the PSD. This apparent exclusion was not only from the immediate vicinity of the postsynaptic membrane but also at nonsynaptic membranes. That this is unlikely to reflect methodological limitations is supported by our results with $\alpha$-actinin; moreover, using the same technique, we can detect other proteins that concentrate close to the membrane and avoid the spine core (Racz et al., 2004). We propose that the spine core represents a previously unrecognized domain playing a special role in cytoskeletal organization and/or spine remodeling.

The present results do not exclude that cortactin may bind to Shank in the PSD. We detected a small amount of cortactin at the cytoplasmic fringe of the PSD, and previous work from our laboratory found Shank to concentrate in the cytoplasmic side of the PSD (Valtschanoff and Weinberg, 2001). Together with previous work (Naisbitt et al., 1999; Husi et al., 2000; Peng et al., 2004), the present data suggest that although a small amount of cortactin lies close to the PSD, it is only weakly associated with it. Thus, unlike the Shank-PSD-95-GKAP (guanylate kinase-associated protein) complex, cortactin is unlikely to be an integral component of the PSD. Our data are consistent with the presence of two distinct pools of cortactin within the spine, a small synaptic pool and a larger core pool, only indirectly coupled to synaptic activity. We speculate that these two pools have distinct functions: the core pool is situated to mediate changes in spine shape, whereas the synaptic pool might help to modify the composition or shape of the PSD in response to specific patterns of activity (Desmond and Levy, 1986; Geinisman et al., 2000; Malinow and Malenka, 2002; Marrone and Petit, 2002; Harris et al., 2003). 

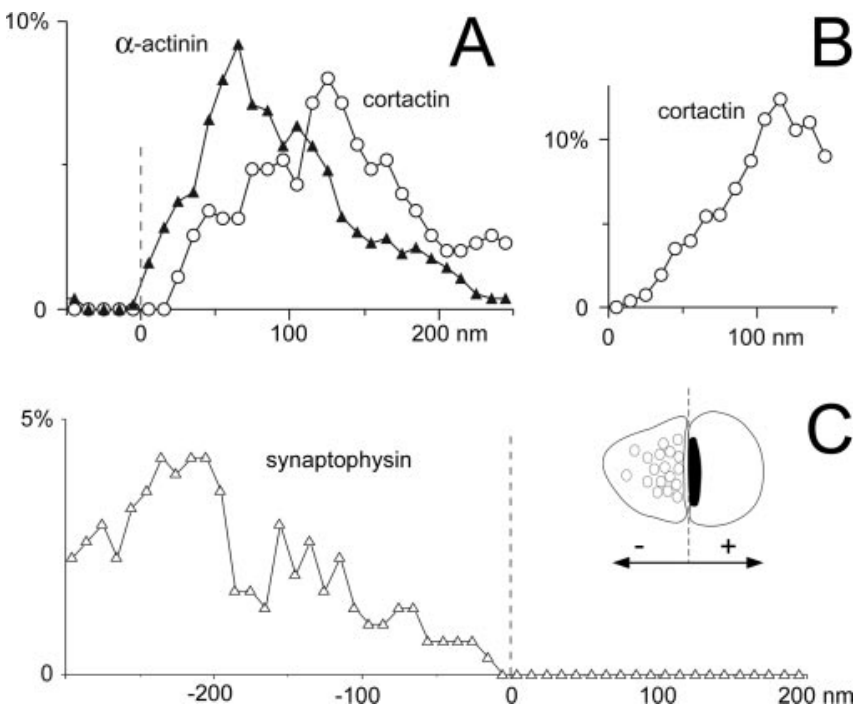

Figure 6. Axodendritic distribution of cortactin, $\alpha$-actinin, and synaptophysin in axospinous synaptic profiles. A, Graph shows the axodendritic distribution of gold particles coding for cor$\operatorname{tactin}(\bigcirc)$ and $\alpha$-actinin $(\mathbf{\Delta})$ in the spine cytoplasm. The $x$-axis values represent the distance from the center of the gold particle to the synaptic membrane, along the axodendritic axis (negative values represent gold particles in the synaptic cleft or presynaptic terminal; see inset in (). The $y$-axis values represent the percentage of the total number of particles counted in each bin. Cortactin lies significantly farther from the PSD than $\alpha$-actinin. $B$, Distances of gold particles coding for cortactin from the spine plasma membrane; cortactin concentrates in the spine core. Only particles lying within the spine were considered; the graph was truncated at $150 \mathrm{~nm}$ to permit inclusion of data from small spines. Labeling density was weighted to compensate for the reduction in perimeter, for particles lying deep in the cytoplasm. C, Graph shows that gold particles coding for synaptophysin $(\triangle)$ are restricted to the presynaptic terminal. The dashed line represents the postsynaptic membrane, which corresponds to 0 in $A$ and $C$ (see inset for explanation). Random photos were taken of spines and terminals in the stratum radiatum of CA1 exhibiting a clear synapse and containing at least one gold particle. Data are from 65 profiles for $\alpha$-actinin, 55 for cortactin, and 55 for synaptophysin. The bin width was $10 \mathrm{~nm}$ for $A$ and $B$ and $20 \mathrm{~nm}$ for $C$. Data were smoothed with a three-point weighted running average.

\section{Cortactin in dendrites}

We found that cortactin is also concentrated in dendritic shafts, although to a lesser degree than in spines. This may represent a transport pool of cortactin en route from the cell body to spines. However, cortactin has also been implicated in the morphogenesis of dendritic trees: $\delta$-catenin can bind to cortactin (Weed et al., 2000); by activating Arp2/3-induced actin polymerization, this catenin-cortactin complex induces process elongation, at least in cultured neurons. In contrast, when $\delta$-catenin (which lies at the plasma membrane) is tyrosine phosphorylated, the dendritic tree becomes highly branched, apparently because the phosphorylation prevents the catenin-cortactin association (Martinez et al., 2003). Thus, our data raise the possibility that cortactin may also play a structural role in mature dendrites.

\section{References}

Ackermann M, Matus A (2003) Activity-induced targeting of profilin and stabilization of dendritic spine morphology. Nat Neurosci 6:1194-1200.

Allison DW, Gelfand VI, Spector I, Craig AM (1998) Role of actin in anchoring postsynaptic receptors in cultured hippocampal neurons: differential attachment of NMDA versus AMPA receptors. J Neurosci 18:2423-2436.

Burette A, Zabel U, Weinberg RJ, Schmidt HH, Valtschanoff JG (2002) Synaptic localization of nitric oxide synthase and soluble guanylyl cyclase in the hippocampus. J Neurosci 22:8961-8970.

Capani F, Martone ME, Deerinck TJ, Ellisman MH (2001a) Selective localization of high concentrations of F-actin in subpopulations of dendritic spines in rat central nervous system: a three-dimensional electron microscopic study. J Comp Neurol 435:156-170.
Capani F, Deerinck TJ, Ellisman MH, Bushong E, Bobik M, Martone ME (2001b) Phalloidin-eosin followed by photo-oxidation: a novel method for localizing F-actin at the light and electron microscopic levels. J Histochem Cytochem 49:1351-1361.

Daly RJ (2004) Cortactin signalling and dynamic actin networks. Biochem J 382:13-25.

Desmond NL, Levy WB (1986) Changes in the postsynaptic density with long-term potentiation in the dentate gyrus. J Comp Neurol 253:476-482.

Fifkova E, Delay RJ (1982) Cytoplasmic actin in neuronal processes as a possible mediator of synaptic plasticity. J Cell Biol 95:345-350.

Fifkova E, Morales M (1992) Actin matrix of dendritic spines, synaptic plasticity, and long-term potentiation. Int Rev Cytol 139:267-307.

Finkel T, Theriot JA, Dise KR, Tomaselli GF, Goldschmidt-Clermont PJ (1994) Dynamic actin structures stabilized by profilin. Proc Natl Acad Sci USA 91:1510-1514.

Fremeau Jr RT, Troyer MD, Pahner I, Nygaard GO, Tran CH, Reimer RJ, Bellocchio EE, Fortin D, Storm-Mathisen J, Edwards RH (2001) The expression of vesicular glutamate transporters defines two classes of excitatory synapse. Neuron 31:247-260.

Fujiyama F, Furuta T, Kaneko T (2001) Immunocytochemical localization of candidates for vesicular glutamate transporters in the rat cerebral cortex. J Comp Neurol 435:379-387.

Fukazawa Y, Saitoh Y, Ozawa F, Ohta Y, Mizuno K, Inokuchi K (2003) Hippocampal LTP is accompanied by enhanced F-actin content within the dendritic spine that is essential for late LTP maintenance in vivo. Neuron 38:447-460.

Geinisman Y, Disterhoft JF, Gundersen HJ, McEchron MD, Persina IS, Power JM, van der Zee EA, West MJ (2000) Remodeling of hippocampal synapses after hippocampus-dependent associative learning. J Comp Neurol 417:49-59.

Halpain S (2000) Actin and the agile spine: how and why do dendritic spines dance? Trends Neurosci 23:141-146.

Harris KM (1999) Structure, development, and plasticity of dendritic spines. Curr Opin Neurobiol 9:343-348.

Harris KM, Fiala JC, Ostroff L (2003) Structural changes at dendritic spine synapses during long-term potentiation. Philos Trans R Soc Lond B Biol Sci 358:745-748.

Hering H, Sheng M (2003) Activity-dependent redistribution and essential role of cortactin in dendritic spine morphogenesis. J Neurosci 23:11759-11769.

Husi H, Ward MA, Choudhary JS, Blackstock WP, Grant SG (2000) Proteomic analysis of NMDA receptor-adhesion protein signaling complexes. Nat Neurosci 3:661-669.

Jahn R, Schiebler W, Ouimet C, Greengard P (1985) A 38,000-dalton membrane protein (p38) present in synaptic vesicles. Proc Natl Acad Sci USA 82:4137-4141.

Kaneko T, Fujiyama F (2002) Complementary distribution of vesicular glutamate transporters in the central nervous system. Neurosci Res 42:243-250.

Kanner SB, Reynolds AB, Vines RR, Parsons JT (1990) Monoclonal antibodies to individual tyrosine-phosphorylated protein substrates of oncogene-encoded tyrosine kinases. Proc Natl Acad Sci USA 87:3328-3332.

Kasai H, Matsuzaki M, Noguchi J, Yasumatsu N, Nakahara H (2003) Structure-stability-function relationships of dendritic spines. Trends Neurosci 26:360-368.

Kennedy MB (2000) Signal-processing machines at the postsynaptic density. Science 290:750-754.

Kim CH, Lisman JE (1999) A role of actin filament in synaptic transmission and long-term potentiation. J Neurosci 19:4314-4324.

Krucker T, Siggins GR, Halpain S (2000) Dynamic actin filaments are required for stable long-term potentiation (LTP) in area CA1 of the hippocampus. Proc Natl Acad Sci USA 97:6856-6861.

Krueger EW, Orth JD, Cao H, McNiven MA (2003) A dynamin-cortactinarp $2 / 3$ complex mediates actin reorganization in growth factorstimulated cells. Mol Biol Cell 14:1085-1096.

Landis DM, Reese TS (1983) Cytoplasmic organization in cerebellar dendritic spines. J Cell Biol 97:1169-1178.

Lorincz A, Notomi T, Tamas G, Shigemoto R, Nusser Z (2002) Polarized and compartment-dependent distribution of HCN1 in pyramidal cell dendrites. Nat Neurosci 5:1185-1193. 
Machesky LM, Insall RH (1998) Scarl and the related Wiskott-Aldrich syndrome protein, WASP, regulate the actin cytoskeleton through the Arp2/3 complex. Curr Biol 8:1347-1356.

Maletic-Savatic M, Malinow R, Svoboda K (1999) Rapid dendritic morphogenesis in CAl hippocampal dendrites induced by synaptic activity. Science 283:1923-1927.

Malinow R, Malenka RC (2002) AMPA receptor trafficking and synaptic plasticity. Annu Rev Neurosci 25:103-126.

Marrone DF, Petit TL (2002) The role of synaptic morphology in neural plasticity: structural interactions underlying synaptic power. Brain Res Brain Res Rev 38:291-308.

Martinez MC, Ochiishi T, Majewski M, Kosik KS (2003) Dual regulation of neuronal morphogenesis by a delta-catenin-cortactin complex and Rho. J Cell Biol 162:99-111.

Matsuzaki M, Honkura N, Ellis-Davies GC, Kasai H (2004) Structural basis of long-term potentiation in single dendritic spines. Nature 429:761-766.

Matus A (2000) Actin-based plasticity in dendritic spines. Science 290:754-758

May RC (2001) The Arp2/3 complex: a central regulator of the actin cytoskeleton. Cell Mol Life Sci 58:1607-1626.

Meyer G, Feldman EL (2002) Signaling mechanisms that regulate actinbased motility processes in the nervous system. J Neurochem 83:490-503.

Mullins RD (2000) How WASP-family proteins and the Arp2/3 complex convert intracellular signals into cytoskeletal structures. Curr Opin Cell Biol 12:91-96.

Naisbitt S, Kim E, Tu JC, Xiao B, Sala C, Valtschanoff J, Weinberg RJ, Worley PF, Sheng M (1999) Shank, a novel family of postsynaptic density proteins that binds to the NMDA receptor/PSD-95/GKAP complex and cortactin. Neuron 23:569-582.

Nikonenko I, Jourdain P, Alberi S, Toni N, Muller D (2002) Activityinduced changes of spine morphology. Hippocampus 12:585-591.

Peng J, Kim MJ, Cheng D, Duong DM, Gygi SP, Sheng M (2004) Semiquantitative proteomic analysis of rat forebrain postsynaptic density fractions by mass spectrometry. J Biol Chem 279:21003-21011.

Racz B, Blanpied TA, Ehlers MD, Weinberg RJ (2004) Lateral organization of endocytic machinery in dendritic spines. Nat Neurosci 7:917-918.

Ramón y Cajal S (1909) Hisotogie du Système Nerveux de l'Homme and des Vertébrés. Madrid: Reprinted by Institute Ramón y Cajal, 1952.

Roelandse M, Welman A, Wagner U, Hagmann J, Matus A (2003) Focal motility determines the geometry of dendritic spines. Neuroscience 121:39-49.

Sala C, Piech V, Wilson NR, Passafaro M, Liu G, Sheng M (2001) Regulation of dendritic spine morphology and synaptic function by Shank and Homer. Neuron 31:115-130.

Segal M, Andersen P (2000) Dendritic spines shaped by synaptic activity. Curr Opin Neurobiol 10:582-586.

Sheng M (2001) Molecular organization of the postsynaptic specialization. Proc Natl Acad Sci USA 98:7058-7061.

Star EN, Kwiatkowski DJ, Murthy VN (2002) Rapid turnover of actin in dendritic spines and its regulation by activity. Nat Neurosci 5:239-246.

Svitkina TM, Borisy GG (1999) Arp2/3 complex and actin depolymerizing factor/cofilin in dendritic organization and treadmilling of actin filament array in lamellipodia. J Cell Biol 145:1009-1026.
Todd AJ, Hughes DI, Polgar E, Nagy GG, Mackie M, Ottersen OP, Maxwell DJ (2003) The expression of vesicular glutamate transporters VGLUT1 and VGLUT2 in neurochemically defined axonal populations in the rat spinal cord with emphasis on the dorsal horn. Eur J Neurosci 17:13-27.

Trachtenberg JT, Chen BE, Knott GW, Feng G, Sanes JR, Welker E, Svoboda $\mathrm{K}$ (2002) Long-term in vivo imaging of experience-dependent synaptic plasticity in adult cortex. Nature 420:788-794.

Tu JC, Xiao B, Naisbitt S, Yuan JP, Petralia RS, Brakeman P, Doan A, Aakalu VK, Lanahan AA, Sheng M, Worley PF (1999) Coupling of mGluR/ Homer and PSD-95 complexes by the Shank family of postsynaptic density proteins. Neuron 23:583-592.

Uruno T, Liu J, Zhang P, Fan Y, Egile C, Li R, Mueller SC, Zhan X (2001) Activation of Arp2/3 complex-mediated actin polymerization by cortactin. Nat Cell Biol 3:259-266.

Uruno T, Liu J, Li Y, Smith N, Zhan X (2003) Sequential interaction of Arp2/3 complex with N-WASP and cortactin during branched actin filament network formation. J Biol Chem 278:26086-26093.

Valtschanoff JG, Weinberg RJ (2001) Laminar organization of the NMDA receptor complex within the postsynaptic density. J Neurosci 21:1211-1217.

Vanderklish PW, Edelman GM (2002) Dendritic spines elongate after stimulation of group 1 metabotropic glutamate receptors in cultured hippocampal neurons. Proc Natl Acad Sci USA 99:1639-1644.

Walikonis RS, Jensen ON, Mann M, Provance DW Jr, Mercer JA, Kennedy MB (2000) Identification of proteins in the postsynaptic density fraction by mass spectrometry. J Neurosci 20:4069-4080.

Weaver AM, Karginov AV, Kinley AW, Weed SA, Li Y, Parsons JT, Cooper JA (2001) Cortactin promotes and stabilizes Arp2/3-induced actin filament network formation. Curr Biol 11:370-374.

Weed SA, Parsons JT (2001) Cortactin: coupling membrane dynamics to cortical actin assembly. Oncogene 20:6418-6434.

Weed SA, Karginov AV, Schafer DA, Weaver AM, Kinley AW, Cooper JA, Parsons JT (2000) Cortactin localization to sites of actin assembly in lamellipodia requires interactions with F-actin and the Arp $2 / 3$ complex. J Cell Biol 151:29-40.

Wiedenmann B, Franke WW (1985) Identification and localization of synaptophysin, an integral membrane glycoprotein of $\mathrm{Mr}$ 38,000 characteristic of presynaptic vesicles. Cell 41:1017-1028.

Wu H, Parsons JT (1993) Cortactin, an 80/85-kilodalton pp60src substrate, is a filamentous actin-binding protein enriched in the cell cortex. J Cell Biol 120:1417-1426.

Wyszynski M, Kharazia V, Shanghvi R, Rao A, Beggs AH, Craig AM, Weinberg R, Sheng M (1998) Differential regional expression and ultrastructural localization of alpha-actinin-2, a putative NMDA receptoranchoring protein, in rat brain. J Neurosci 18:1383-1392.

Yoshimura Y, Yamauchi Y, Shinkawa T, Taoka M, Donai H, Takahashi N, Isobe T, Yamauchi T (2004) Molecular constituents of the postsynaptic density fraction revealed by proteomic analysis using multidimensional liquid chromatography-tandem mass spectrometry. J Neurochem 88:759-768.

Yuste R, Bonhoeffer T (2001) Morphological changes in dendritic spines associated with long-term synaptic plasticity. Annu Rev Neurosci 24:1071-1089. 